\title{
BRST extension of the Faddeev model
}

\author{
Soon-Tae Hong* \\ Department of Science Education, Ewha Womans University, Seoul 120-750, Korea and \\ Department of Physics, University of Tokyo, Tokyo 113-0033, Japan \\ Antti J. Niemi ${ }^{\dagger}$ \\ Department of Theoretical Physics, Uppsala University, P.O. Box 803, S-75108 Uppsala, Sweden and \\ Department of Physics, University of Tokyo, Tokyo 113-0033, Japan
}

(Dated: November 5, 2018)

\begin{abstract}
The Faddeev model is a second class constrained system. Here we construct its nilpotent BRST operator and derive the ensuing manifestly BRST invariant Lagrangian. Our construction employs the structure of Stückelberg fields in a nontrivial fashion.
\end{abstract}

PACS numbers: 11.10.Ef; 11.10.Lm; 11.30.-j

Keywords: Hamiltonian approach; nonlinear model; BRST symmetry; ghost field

The Faddeev model is a natural extension of the Heisenberg $\mathrm{O}(3)$-model. It appears in many physical applications from high energy physics [1] to condensed matter physics [2], and its prominent feature is the presence of knotted solitons [3]. The model is defined by the Lagrangian [4],

$$
L_{0}=\int d^{3} x\left[m^{2}\left(\partial_{\mu} n^{a}\right)\left(\partial^{\mu} n^{a}\right)+\frac{1}{e^{2}} H_{\mu \nu} H^{\mu \nu}\right] .
$$

Here $m$ is a mass scale and $e$ is a dimensionless coupling constant, and $H_{\mu \nu}$ are defined as

$$
H_{\mu \nu}=\epsilon_{a b c} n^{a} \partial_{\mu} n^{b} \partial_{\nu} n^{c},
$$

where the components $n^{a}(a=1,2,3)$ define a vector field with unit length. Since time derivatives appear in (1) at most quadratically, the Faddeev model allows for a Hamiltonian interpretation. But due to the condition

$$
n^{a} n^{a}-1=0 .
$$

it is a second class constrained Hamiltonian system. In order to maintain manifest Lorentz invariance in the Hamiltonian formalism, we then need to resort to an appropriate extension of the Hamiltonian Becchi-Rouet-Stora-Tyutin (BRST) formalism [5].

We start by interpreting the Lagrangian (1) in terms of its Hamiltonian variables. From (1), we find that the canonical momenta conjugate to the real scalar fields $n^{a}$ are given by

$$
\pi^{a}=\frac{\delta L_{0}}{\delta \partial_{0} n^{a}}=2 m^{2} \partial_{0} n^{a}-\frac{4}{e^{2}} A_{i}^{a} A_{i}^{b} \partial_{0} n^{b},
$$

where the $A_{i}^{a}$ are

$$
A_{i}^{a}=\epsilon_{a b c} n^{c} \partial_{i} n^{b}
$$

From (2) we can then solve for the time derivative $\partial_{0} n^{a}$ in terms of the canonical momenta $\pi^{a}$. The result can be expressed in terms of a power series in $1 / e^{2}$, and the first two terms are

$$
\partial_{0} n^{a}=\frac{2}{m^{2}} \pi^{a}+\frac{1}{m^{4} e^{2}} A_{i}^{a} A_{i}^{b} \pi^{b}+O\left(\frac{1}{e^{4}}\right) .
$$

This leads to the canonical Hamiltonian

$$
H=\int d^{3} x\left[\frac{1}{4 m^{2}} \pi^{a} \pi^{a}+m^{2}\left(\partial_{i} n^{a}\right)\left(\partial_{i} n^{a}\right)-\frac{1}{e^{2}} H_{i j}^{2}+\frac{1}{2 m^{4} e^{2}} A_{i}^{a} A_{i}^{b} \pi^{a} \pi^{b}\right]+O\left(\frac{1}{e^{4}}\right),
$$

\footnotetext{
*Electronic address: soonhong@ewha.ac.kr

${ }^{\dagger}$ Electronic address: niemi@teorfys.uu.se
} 
where the canonical variables are subject to the Poisson bracket

$$
\left\{n^{a}(x), \pi^{b}(y)\right\}=\delta^{a b} \delta^{3}(x-y) .
$$

By implementing the Dirac algorithm [6] we conclude that together with the identity

$$
A_{i}^{a} n^{a}=0
$$

our Hamiltonian system is subject to the following second class constraints

$$
\begin{aligned}
& \Omega_{1}=n^{a} n^{a}-1 \approx 0, \\
& \Omega_{2}=n^{a} \pi^{a} \approx 0 .
\end{aligned}
$$

With $\epsilon^{12}=-\epsilon^{21}=1$ this second class constraint algebra is

$$
\Delta_{k k^{\prime}}(x, y)=\left\{\Omega_{k}(x), \Omega_{k^{\prime}}(y)\right\}=\epsilon^{k k^{\prime}} n^{a} n^{a} \delta^{3}(x-y) .
$$

Following the Hamiltonian quantization scheme for constrained systems $[6,7,8,9,10]$ we proceed to convert the second class constraints $\Omega_{i}=0(i=1,2)$ into first class ones. For this we introduce two canonically conjugate Stückelberg fields $\left(\theta, \pi_{\theta}\right)$ with Poisson bracket

$$
\left\{\theta(x), \pi_{\theta}(y)\right\}=\delta^{3}(x-y) .
$$

The strongly involutive first class constraints $\tilde{\Omega}_{i}$ are constructed as a power series of the Stückelberg fields, and the result is

$$
\begin{aligned}
& \tilde{\Omega}_{1}=\Omega_{1}+2 \theta, \\
& \tilde{\Omega}_{2}=\Omega_{2}-n^{a} n^{a} \pi_{\theta} .
\end{aligned}
$$

We proceed to the construction of first class canonical variables $\tilde{\mathcal{F}}=\left(\tilde{n}^{a}, \tilde{\pi}^{a}\right)$, that correspond to the original variables $\mathcal{F}=\left(n^{a}, \pi^{a}\right)$ in the extended phase space. These variables are obtained as a power series in the Stückelberg fields $\left(\theta, \pi_{\theta}\right)$, by demanding that they are in strong involution with the first class constraints $(5)$, that is

$$
\left\{\tilde{\Omega}_{i}, \tilde{\mathcal{F}}\right\}=0 .
$$

After some straightforward but tedious algebra, we obtain for the first class canonical variables

$$
\begin{aligned}
\tilde{n}^{a} & =n^{a}\left(\frac{n^{c} n^{c}+2 \theta}{n^{c} n^{c}}\right)^{1 / 2}, \\
\tilde{\pi}^{a} & =\left(\pi^{a}-n^{a} \pi_{\theta}\right)\left(\frac{n^{c} n^{c}}{n^{c} n^{c}+2 \theta}\right)^{1 / 2}, \\
\tilde{H}_{i j} & =\epsilon_{a b c} n^{a} \partial_{i} n^{b} \partial_{j} n^{c}\left(\frac{n^{d} n^{d}+2 \theta}{n^{d} n^{d}}\right)^{3 / 2}, \\
\tilde{A}_{i}^{a} & =\epsilon_{a b c} n^{c} \partial_{i} n^{b} \frac{n^{d} n^{d}+2 \theta}{n^{d} n^{d}} .
\end{aligned}
$$

Note in particular that now these first class variables are not truncated but exact, unlike in the case of the explicit Hamiltonian that we have displayed in (3).

In terms of the first class variables we obtain for the (exact) Hamiltonian

$$
\tilde{H}=\int d^{3} x\left[\frac{1}{4 m^{2}} \tilde{\pi}^{a} \tilde{\pi}^{a}+m^{2}\left(\partial_{i} \tilde{n}^{a}\right)\left(\partial_{i} \tilde{n}^{a}\right)-\frac{1}{e^{2}} \tilde{H}_{i j}^{2}+\frac{1}{2 m^{4} e^{2}} \tilde{A}_{i}^{a} \tilde{A}_{i}^{b} \tilde{\pi}^{a} \tilde{\pi}^{b}\right] .
$$

Explicitly, in terms of the original fields

$$
\begin{aligned}
\tilde{H}= & \int d^{3} x\left[\frac{1}{4 m^{2}}\left(\pi^{a}-n^{a} \pi_{\theta}\right)\left(\pi^{a}-n^{a} \pi_{\theta}\right) \frac{n^{c} n^{c}}{n^{c} n^{c}+2 \theta}+m^{2}\left(\partial_{i} n^{a}\right)\left(\partial_{i} n^{a}\right) \frac{n^{c} n^{c}+2 \theta}{n^{c} n^{c}}\right. \\
& \left.-\frac{1}{e^{2}} H_{i j}^{2}\left(\frac{n^{c} n^{c}+2 \theta}{n^{c} n^{c}}\right)^{3}+\frac{1}{2 m^{4} e^{2}} A_{i}^{a} A_{i}^{b}\left(\pi^{a}-n^{a} \pi_{\theta}\right)\left(\pi^{b}-n^{b} \pi_{\theta}\right) \frac{n^{c} n^{c}+2 \theta}{n^{c} n^{c}}\right] .
\end{aligned}
$$


Notice that this Hamiltonian is strongly involutive with the first class constraints,

$$
\left\{\tilde{\Omega}_{i}, \tilde{H}\right\}=0 .
$$

Note also that the first class constraints (5) can be rewritten as

$$
\begin{aligned}
& \tilde{\Omega}_{1}=\tilde{n}^{a} \tilde{n}^{a}-1, \\
& \tilde{\Omega}_{2}=\tilde{n}^{a} \tilde{\pi}^{a} .
\end{aligned}
$$

These have the same functional form as the second class constraints (4) but now we have the first class constraint algebra

$$
\left\{\tilde{\Omega}_{i}, \tilde{\Omega}_{j}\right\}=0 .
$$

However, when we now consider the time evolution of the constraint algebra, as determined by computing the Poisson brackets of the constraints with the Hamiltonian (6), we conclude from the Poisson bracket

$$
\left\{\tilde{\Omega}_{1}, \tilde{H}\right\}=0,
$$

that there is a need to improve the Hamiltonian into the following, equivalent first class Hamiltonian,

$$
\tilde{H}^{\prime}=\tilde{H}+\int d^{3} x \frac{1}{2 m^{2}} \pi_{\theta} \tilde{\Omega}_{2} .
$$

Indeed, this improved Hamiltonian generates the constraint algebra

$$
\begin{aligned}
\left\{\tilde{\Omega}_{1}, \tilde{H}^{\prime}\right\} & =\frac{1}{m^{2}} \tilde{\Omega}_{2}, \\
\left\{\tilde{\Omega}_{2}, \tilde{H}^{\prime}\right\} & =0 .
\end{aligned}
$$

Obviously, since the Hamiltonians $\tilde{H}$ and $\tilde{H}^{\prime}$ only differ by a term which vanishes on the constraint surface, they lead to an equivalent dynamics on the constraint surface.

We now proceed to the implementation of the covariant Batalin-Fradkin-Vilkovisky (BFV) formalism [11]. We start by the construction of the nilpotent BRST operator. For this, we introduce two canonical sets of ghost and anti-ghost fields, together with auxiliary fields $\left(\mathcal{C}^{i}, \overline{\mathcal{P}}_{i}\right),\left(\mathcal{P}^{i}, \overline{\mathcal{C}}_{i}\right),\left(N^{i}, B_{i}\right)(i=1,2)$. The BRST operator for our constraint algebra is then simply

$$
Q=\int \mathrm{d}^{3} x\left(\mathcal{C}^{i} \tilde{\Omega}_{i}+\mathcal{P}^{i} B_{i}\right)
$$

We choose the unitary gauge with

$$
\chi^{1}=\Omega_{1}, \quad \chi^{2}=\Omega_{2}
$$

by selecting the gauge fixing functional

$$
\Psi=\int \mathrm{d}^{3} x\left(\overline{\mathcal{C}}_{i} \chi^{i}+\overline{\mathcal{P}}_{i} N^{i}\right)
$$

Clearly,

$$
Q^{2}=\{Q, Q\}=0,
$$

and explicitly $Q$ is the generator of the following infinitesimal transformations

$$
\begin{array}{ll}
\delta_{Q} n^{a}=-\mathcal{C}^{2} n^{a}, & \delta_{Q} \pi^{a}=2 \mathcal{C}^{1} n^{a}+\mathcal{C}^{2}\left(\pi^{a}-2 n^{a} \pi_{\theta}\right), \\
\delta_{Q} \theta=\mathcal{C}^{2} n^{a} n^{a}, & \delta_{Q} \pi_{\theta}=2 \mathcal{C}^{1} \\
\delta_{Q} \mathcal{C}^{i}=0, & \delta_{Q} \overline{\mathcal{P}}_{i}=\tilde{\Omega}_{i}, \\
\delta_{Q} \mathcal{P}^{i}=0, & \delta_{Q} \overline{\mathcal{C}}_{i}=B_{i}, \\
\delta_{Q} N^{i}=-\mathcal{P}^{i}, & \delta_{Q} B_{i}=0
\end{array}
$$


Furthermore, we have

$$
\begin{aligned}
\delta_{Q} \tilde{H} & =\{Q, \tilde{H}\}=0, \\
\delta_{Q}\{Q, \Psi\} & =\{Q,\{Q, \Psi\}\}=0,
\end{aligned}
$$

where the second line follows from the nilpotentcy of the charge $Q$. The "gauge fixed" BRST invariant Hamiltonian is now given by

$$
H_{e f f}=\tilde{H}-\{Q, \Psi\}
$$

with $\tilde{H}$ defined in (6). It is clearly BRST invariant.

After some algebra which is associated with the evaluation of the Legendre transformation of $H_{e f f}$, we arrive at the following manifestly covariant BRST improved (quantum) Lagrangian

$$
L_{e f f}=L_{0}+L_{W Z}+L_{g h o s t}
$$

where $L_{0}$ is given by $(1)$ and

$$
\begin{aligned}
L_{W Z} & =\int d^{3} x\left[\frac{2 m^{2}}{n^{c} n^{c}}\left(\partial_{\mu} n^{a}\right)\left(\partial^{\mu} n^{a}\right) \theta+\frac{1}{e^{2}} H_{\mu \nu} H^{\mu \nu}\left(3+\frac{6 \theta}{n^{c} n^{c}}+\frac{4 \theta^{2}}{\left(n^{c} n^{c}\right)^{2}}\right)-\frac{m^{2}}{\left(n^{c} n^{c}\right)^{2}} \partial_{\mu} \theta \partial^{\mu} \theta\right], \\
L_{\text {ghost }} & =\int d^{3} x\left[-m^{2}\left(n^{a} n^{a}\right)^{2}\left(B_{2}+2 \overline{\mathcal{C}}_{2} \mathcal{C}^{2}\right)^{2}-\frac{1}{n^{c} n^{c}} \partial_{\mu} \theta \partial^{\mu} B_{2}+\partial_{\mu} \overline{\mathcal{C}}_{2} \partial^{\mu} \mathcal{C}^{2}\right] .
\end{aligned}
$$

This is our main result, a manifestly covariant version of the Faddeev model (1) where the variable $n^{a}$ is now an unconstrained variable. Note that in deriving (8) we have included all the higher order terms of $1 / e^{2}$, that we truncated in displaying (3). We also note that the (BRST gauge fixed) effective Lagrangian (8) is manifestly invariant under the following (Lagrangian) BRST transformation,

$$
\begin{array}{ll}
\delta_{\epsilon} n^{a}=\epsilon n^{a} \mathcal{C}^{2}, & \delta_{\epsilon} \theta=-\epsilon n^{a} n^{a} \mathcal{C}^{2} \\
\delta_{\epsilon} \overline{\mathcal{C}}_{2}=-\epsilon B_{2}, & \delta_{\epsilon} \mathcal{C}^{2}=\delta_{\epsilon} B_{2}=0
\end{array}
$$

where $\epsilon$ is an infinitesimal Grassmann valued parameter. Finally, we note that the Stückelberg field $\theta$ becomes a nontrivial, propagating field degree of freedom.

In conclusion, we have derived the BRST improved version of the Faddeev model. It has the advantage, that the explicit implementation of the second class constraint which enforces the order parameter $n^{a}$ to be normalized into unity, can be avoided. In order to obtain the BRST version of the Faddeev model, we have first employed the Stückelberg fields to convert the second class constraint algebra into a first class algebra. In particular, the Stückelberg fields appear in a nontrivial manner in the Lagrangian BRST version of the Faddeev model.

Acknowledgments The authors would like to thank Tohru Eguchi for warm hospitality at University of Tokyo, where this work was completed. S.T.H. would like to acknowledge financial support in part from the Korea Science and Engineering Foundation Grant R01-2000-00015. The research by A.N. has been supported by a grant from VR (Vetenskapsrädet) and by the STINT Thunberg Fellowship.

[1] L.D. Faddeev and A.J. Niemi, Phys. Rev. Lett. 82, 1624 (1999).

[2] E. Babaev, L.D. Faddeev and A.J. Niemi, Phys. Rev. B65, 100512 (2002).

[3] L.D. Faddeev and A.J. Niemi, Nature 387, 58 (1997).

[4] L.D. Faddeev, Princeton Report No. IAS-75-QS70 (1970).

[5] C. Becchi, A. Rouet and R. Stora, Ann. Phys. 98, 287 (1976); I.V. Tyutin, Lebedev Preprint 39 (1975) unpublished.

[6] P.A.M. Dirac, Lectures on Quantum Mechanics (Yeshiba University Press, New York, 1964).

[7] L.D. Faddeev and S.L. Shatashvili, Phys. Lett. B167, 225 (1986).

[8] I.A. Batalin and E.S. Fradkin, Phys. Lett. B180, 157 (1986); I.A. Batalin and E.S. Fradkin, Nucl. Phys. B279, 514 (1987); I.A. Batalin and I.V. Tyutin, Int. J. Mod. Phys. A6, 3255 (1991).

[9] A.J. Niemi, Phys. Lett. B213, 41 (1988).

[10] S.T. Hong and Y.J. Park, Phys. Rep. 358, 143 (2002), and references therein.

[11] E.S. Fradkin and G.A. Vilkovisky, Phys. Lett. B55, 224 (1975); M. Henneaux, Phys. Rep. 126, 1 (1985). 